\title{
EXTENSIONS OF A THEOREM OF FUGLEDE AND PUTNAM
}

\author{
S. K. BERBERIAN
}

\begin{abstract}
The operator equation $A X=X B$ implies $A^{*} X=X B^{*}$ when $A$ and $B$ are normal (theorem of Fuglede and Putnam). If $X$ is of HilbertSchmidt class, the assumptions on $A$ and $B$ can be relaxed: it suffices that $A$ and $B^{*}$ be hyponormal, or that $B$ be invertible with $\|A\|\left\|B^{-1}\right\|<1$.
\end{abstract}

The classical Fuglede-Putnam theorem asserts that if $A, B, X$ are operators in a Hilbert space such that $A X=X B$, and if $A$ and $B$ are normal, then also $A^{*} X=X B^{*}$ [4, Problem 152]. In this note we relax the hypotheses on $A$ and $B$, at the cost of requiring $X$ to be of Hilbert-Schmidt class. The resulting extensions of the Fuglede-Putnam theorem are perhaps unexciting, although it is somewhat surprising that normality can be dropped; of possibly greater interest is the curious method of proof. Our theorem is as follows:

Theorem. Suppose $A, B, X$ are operators in the Hilbert space $H$, such that

$$
A X=X B \text {. }
$$

Assume also that $X$ is an operator of Hilbert-Schmidt class. Then

$$
A^{*} X=X B^{*}
$$

under either of the following hypotheses: (i) $A$ and $B^{*}$ are hyponormal; (ii) $B$ is invertible and $\|A\|\left\|B^{-1}\right\| \leqslant 1$.

The proof employs what are essentially tensor product techniques. The operators in $H$ of Hilbert-Schmidt class form an ideal $\mathcal{H}$ in the algebra $\mathcal{E}(H)$ of all operators in $H$, and $\mathcal{H}$ is itself a Hilbert space for the inner product

$$
(X \mid Y)=\sum\left(X e_{\iota} \mid Y e_{\iota}\right)=\operatorname{Tr}\left(Y^{*} X\right)=\operatorname{Tr}\left(X Y^{*}\right),
$$

where $\left(e_{\iota}\right)$ is any orthonormal basis of $H\left[3\right.$, Chapter I, $\S 6, \mathrm{n}^{0} 6$, Corollary of Theorem 5]. (One could identify $\mathcal{H}$ with $\bar{H} \otimes H$, where $\bar{H}$, awkwardly, is the conjugate Hilbert space of $H$; it seems simpler to work directly with $\mathcal{H}$.)

For each pair of operators $A, B \in \mathcal{L}(H)$, there is defined an operator $\mathcal{T} \in \mathcal{L}(\mathcal{H})$ via the formula $\mathcal{T} X=A X B$ (it is suggestive, though not strictly correct, to view $\mathcal{T}$ as the operator $A \otimes B$ ). Evidently $\|\mathcal{T}\| \leqslant\|A\|\|B\|$ (in fact equality holds, but we do not need it). The adjoint of $\mathcal{T}$ is given by the formula $\mathcal{T}^{*} X=A^{*} X B^{*}$, as one sees from the calculation $\left(\mathcal{T}^{*} X \mid Y\right)=$ $(X \mid \mathcal{T} Y)=(X \mid A Y B)=\operatorname{Tr}\left(X B^{*} Y^{*} A^{*}\right)=\operatorname{Tr}\left(A^{*} X B^{*} Y^{*}\right)=\left(A^{*} X B^{*} \mid Y\right)$. It follows at once that if $A$ and $B$ are both normal (resp. both selfadjoint), then

Received by the editors June 2, 1977.

AMS (MOS) subject classifications (1970). Primary 47A50; Secondary 47B20. 
so is $\mathcal{T}$ (more precisely, see the lemma below). If $A \geqslant 0$ and $B \geqslant 0$, then also $\sigma \geqslant 0$, as one sees from the calculation $(\tau X \mid X)=\operatorname{Tr}\left(A X B X^{*}\right)=$ $\operatorname{Tr}\left(A^{1 / 2} X B X^{*} A^{1 / 2}\right)=\operatorname{Tr}\left[\left(A^{1 / 2} X B^{1 / 2}\right)\left(A^{1 / 2} X B^{1 / 2}\right)^{*}\right] \geqslant 0 ;$ indeed, $\mathscr{T}^{1 / 2} X=$ $A^{1 / 2} X B^{1 / 2}$.

LEMMA. If $A$ and $B^{*}$ are hyponormal, then the operator $\mathcal{T}$ in $\mathcal{H}$ defined by $\sigma X=A X B$ is also hyponormal.

Proof. We are assuming that $A A^{*} \leqslant A^{*} A$ and $B^{*} B \leqslant B B^{*}$, and we must show that $\mathcal{T} \mathcal{J}^{*} \leqslant \mathcal{T}^{*} \mathcal{T}$. Indeed, the formula

$$
\begin{aligned}
\left(\mathcal{\sigma} * \sigma-\sigma \mathcal{T}^{*}\right) X & =A^{*} A X B B^{*}-A A^{*} X B^{*} B \\
& =\left(A^{*} A-A A^{*}\right) X B B^{*}+A A^{*} X\left(B B^{*}-B^{*} B\right)
\end{aligned}
$$

shows that $\mathcal{T} * \mathcal{T}-\mathcal{T} \mathcal{T} *$ is the sum of two positive operators.

Proof of THE THEOREM. (i) Suppose first that, in addition to (i), $B$ is invertible. Let $\mathcal{T}$ be the operator in $\mathcal{H}$ defined by $\mathcal{T} Y=A Y B^{-1}$; since $A$ and $\left(B^{-1}\right)^{*}=\left(B^{*}\right)^{-1}$ are hyponormal [2, Chapter VI, §9, Exercise 11], it follows from the lemma that $\mathcal{T}$ is hyponormal. Since $\mathcal{} T=X$ by (1), it follows that $\mathcal{T}^{*} X=X\left[2\right.$, Chapter VII, $\S 3$, Exercise 5(i)], that is, $A^{*} X\left(B^{-1}\right)^{*}=X$, whence (2). In the general case, choose a complex number $\lambda$ such that $B-\lambda$ is invertible, and apply the preceding argument to $A-\lambda, B-\lambda$ in place of $A, B$.

(ii) The operator $\mathcal{T}$ in $\mathcal{H}$ defined by $\mathcal{T} Y=A Y B^{-1}$ satisfies $\|\mathcal{T}\| \leqslant 1$ by (ii), and $\mathcal{} T=X$ by (1), therefore $\mathcal{T}^{*} X=X[5, \S 143]$, whence (2). (Incidentally, instead of (ii) one could suppose that for some $\lambda, B-\lambda$ is invertible and $\|A-\lambda\|\left\|(B-\lambda)^{-1}\right\| \leqslant 1$; or that $A$ is invertible and $\left\|A^{-1}\right\|\|B\| \leqslant 1$.)

If $H$ is finite-dimensional, then every operator is of Hilbert-Schmidt class and every hyponormal operator is normal [2, Chapter VII, §2, Exercise 6], thus the assertion of case (i) of the theorem is the Fuglede-Putnam theorem for the finite-dimensional case; there is an even simpler trace argument for this case due to I. Kaplansky (cf. [1, Theorem 4]).

AdDendum. Part (i) of the theorem is related to a question raised by J. G. Stampfli and B. L. Wadhwa [An asymmetric Putnam-Fuglede theorem for dominant operators, Indiana Univ. Math. J. 25 (1976), 359-365, Remark following Theorem 3]. The idea of (ii) is like that of Theorem 5.1 of the paper of C. Davis and W. M. Kahan [The rotation of eigenvectors by a perturbation. III, SIAM J. Numer. Anal. 7 (1970), 1-46; MR 41 \#9044]. I am indebted to the referee for these remarks.

\section{REFERENCES}

1. S. K. Berberian, Note on a theorem of Fuglede and Putnam, Proc. Amer. Math. Soc. 10 (1959), 175-182.

2. Introduction to Hilbert space, Chelsea, New York, 1976.

3. J. Dixmier, Les algèbres d'opérateurs dans l'espace hilbertien (Algèbres de von Neumann), 2nd ed., Gauthier-Villars, Paris, 1969.

4. P. R. Halmos, A Hilbert space problem book, Springer-Verlag, New York, 1974.

5. F. Riesz and B. Sz.-Nagy, Lecons d'analyse fonctionelle, Akadémia Kiadó, Budapest, 1952.

Department of Mathematics, University of TeXas, Austin, TeXas 78712 